\title{
The Role of Nuclear Medicine in Malignant Melanoma
}

Anna N. Paschali*

Clinical Research Fellow, University of Patras, Medical School, Greece

*Corresponding author: Anna N. Paschali, Nuclear Medicine Physician, Clinical Research Fellow, University of Patras, Medical School, Greece, Tel: +30 2610 999210; E-mail: apashali@upatras.gr

Received date: July 08, 2015, Accepted date: July 27, 2015, Publication date: July 30, 2015

Copyright: @ 2015 Paschali AN. This is an open-access article distributed under the terms of the Creative Commons Attribution License; which permits unrestricted use; distribution; and reproduction in any medium; provided the original author and source are credited.

\begin{abstract}
Malignant Melanoma (MM) accounts only for $4 \%$ of all skin cancers, but is the most aggressive and lethal type of skin cancer. MM metastizes either to regional lymph nodes or to distant sites following predictable or unpredictable pathways. Nuclear Medicine (NM) plays a key role in nodal staging with the use of sentinel lymph node (SLN) mapping and guided sampling techniques. ${ }^{18} \mathrm{~F}$-Fluorodeoxyglucose ( ${ }^{18} \mathrm{~F}$-FDG) Positron Emission Tomography $(\mathrm{PET})$ has also a pivotal role in staging and restaging of $\mathrm{MM}$ and can alter significantly patient's management. In today's clinical practice, hybrid SPECT/CT and PET/CT scanners allow simultaneous assessment of both metabolic and anatomic characteristics of the primary tumor and its potential local, regional, and distant extension. More studies are required to determine the optimal surveillance schedules of PET/CT in patients with high-risk MM in order to maximize the detection of early relapse, as well as the role of PET/CT in the assessment of response to MM therapy.
\end{abstract}

In this paper, we review the clinical contribution of current nuclear medicine technologies to the management of MM.

Keywords: Nuclear medicine; PET/CT; SPECT/CT; SLN; Malignant melanoma

\section{Nuclear Medicine}

Nuclear imaging is based on the injection of radionuclides in their native form or as a part of a chemical molecule following the tracer principle, which basically means that very minute amounts of active substances are used without disturbing vital biological processes. Thus, the body is imaged "from the inside out" using special devices (PET or $\gamma$-camera) which record the distribution of radioactivity from administered radionuclides. The strengths of nuclear imaging are its high sensitivity, the increased number of clinical scanners installed and a wide variety of targeted, radioactively labeled tracers in clinical applications. Nuclear medicine techniques, such as sentinel lymph node (SLN) mapping and ${ }^{18} \mathrm{~F}$-fluorodeoxyglucose (18F-FDG) Positron Emission Tomography (PET), have played key roles in nodal and distant staging of malignant melanoma (MM).

\section{F-FDG PET}

PET is a nuclear imaging technique that uses short-lived isotopes (positron emitters) such as fluorine-18 $\left({ }^{18} \mathrm{~F}, \mathrm{~T}_{1 / 2}=110 \mathrm{~min}\right)$ for labelling of biomolecules for in-vivo characterization of physiologic and biochemical processes. The positron emitted travels a short distance, determined by its initial kinetic energy, before it meets an electron and undergoes the annihilation reaction. Upon annihilation, the mass of positron and electron is converted into two $511 \mathrm{keV}$ photons that travel in approximately opposite directions. Coincidence detection of these annihilation photons followed by mathematical reconstruction of the images form the basis of PET imaging. The inherent resolution of PET system is fundamentally limited by physical behavior of positron decay, associated with the significant movement of positron before annihilation, and deviation from exact $180^{\circ}$ angular separation [1]. Current state-of-the-art clinical PET scanners have a spatial resolution of 3-6 $\mathrm{mm}$. A major limitation for the widespread use of PET remains the high cost, reflecting the sophisticated imaging technology and the need of a cyclotron on-site or in close proximity for production of short-lived tracers. The relatively long half life of $18 \mathrm{~F}$ makes it suitable for imaging in centres without access to an on-site cyclotron.

The most frequently used biomolecule in oncology is fluorodeoxyglucose (FDG) labeled with $18 \mathrm{~F}\left({ }^{18} \mathrm{~F}-\mathrm{FDG}\right)$, a glucose analog taken up into cells via glucose transporters (GLUTs). Similar to glucose, $18 \mathrm{~F}-\mathrm{FDG}$ is then phosphorylated by hexokinase to ${ }^{18} \mathrm{~F}$ FDG-6-phosphate but unlike glucose-6-phosphate, subsequent metabolism of ${ }^{18} \mathrm{~F}-\mathrm{FDG}$-6-phosphate is minimal so that ${ }^{18} \mathrm{~F}$-FDG becomes essentially trapped into the cells. Malignant cells compared to normal cells, over-express glucose transporters and have increased intracellular enzyme levels of hexokinase while reduced levels of glucose-6-phosphatase (an enzyme which metabolizes FDG-6phosphate). FDG provides information on glucose metabolism of tissue (metabolic imaging) but is not a cancer specific agent, as it is also taken by inflammatory cells (especially activated macrophages) that present an increased glycolytic rate.

Contemporary PET systems are combined with multi detector computerized tomography of low or full dose (PET/CT), providing a combination of metabolic and anatomic information and correction of emission data, resulting in superior image quality. It is widely accepted that PET/CT appears to be superior to stand-alone PET and in our days only hybrid PET/CT systems are installed in hospitals worldwide.

Hybrid PET and magnetic resonance (PET/MR) scanners have recently introduced and create high expectations, notably because of the potential for superior tissue contrast inherent in the MR modality, as well as the potential for multiparametric functional imaging in conjunction with PET. Access to co-registered, almost simultaneous 
Page 2 of 8

anatomic, physiologic and molecular measurements have already made PET/MR the most sophisticated quantitative imaging modality, although these applications are in their infancy [2].

In translational research, ${ }^{18} \mathrm{~F}$-FDG-sensitive intra-operative probes are being designed to detect small nodal and visceral metastases of $\mathrm{MM}$ and may become important tools for the cancer surgeon, if this technique proves its value in clinical trials.

\section{SPECT}

Single photon emission computed tomography (SPECT) is another nuclear medicine technique that uses $\gamma$-emitting radionuclides, such as technetium- $99 \mathrm{~m} \quad{ }^{99 \mathrm{~m}} \mathrm{Tc}, \mathrm{T}_{1 / 2}=6$ hours) for labelling of specific molecules. SPECT is performed in the conventional $\gamma$-camera (single or multi head) from the reconstruction of multiple static projections from different angles around the body. The spatial resolution of clinical SPECT systems is $7-15 \mathrm{~mm} .{ }^{99 \mathrm{~m}}$ Tc labeled tracers are the most readily available in every day clinical practice from a commercially available molybdenum generator system. ${ }^{99 \mathrm{~m}} \mathrm{Tc}$ has also favorable physical and dosimetric features as it decays by isomeric transition and emits gamma rays at an energy level of $140 \mathrm{keV}$, and is the preferred isotope for SPECT imaging.

For SLN mapping the radiopharmaceuticals used encompass a variety of colloid particles labeled with ${ }^{99 \mathrm{~m}} \mathrm{Tc}$ (radiocolloid), that are taken up by macrophages in lymph nodes. It has been suggested that the optimal particle size for SLN detection is 100-200 $\mathrm{nm}$ [3].

Bone scintigraphy using ${ }^{99} \mathrm{~m} \mathrm{Tc}$ labeled biphosphonates is a sensitive and widely available screening tool for metastases to the whole skeleton, although osseous metastases from MM tend to be lytic, and hence more difficult to detect on bone scintigraphy.

The recent introduction of hybrid SPECT/CT systems consisting of multi detector CT (low or full dose) and a $\gamma$-camera combined in the same gantry enables transmission (CT) and emission (SPECT) procedures to be performed without changing patient position, with subsequent automatic fusion of images from both modalities.

\section{Radionuclide sentinel lymph node (SLN) mapping and directed sampling technique}

Sentinel lymph node(s) (SLNs) node is (are) the first node(s) to drain the primary tumor and is the most possible to contain metastasis. Metastatic spread to lymph nodes follows the lymphatic drainage pattern of the regional lymph node basin(s) draining the skin of the site of the primary MM. These drainage patterns are largely predictable for the extremities, but head and especially torso lesions can have multiple possible draining lymph node basins due to the superficial drainage routes of cutaneous MM.

Radiotracer SLN mapping is performed preoperarively, after intradermal or subcutaneous injection of radiocolloid around the site of the primary tumor in multiple (2-4) small volume injections. Immediately after the injections, dynamic images are acquired (dynamic lymphoscintigraphy) to image the lymphatic channels and indicate the number of nodes on a direct drainage pathway and distinguish these from second-tier nodes. Dynamic study is followed by multiple static planar images of the draining lymph node basins until the SLNs to be visualized (Chakera $\mathrm{AH}$ ).

A dual-headed camera can be advantageous in allowing different views simultaneously during the imaging phase. More accurate localization of SLNs before surgery is achievable with the new hybrid SPECT/CT systems. The clinical information added by SPECT/CT is valuable when MM lesion is in body parts with complex lymphatic drainage systems, such as the head and neck and the posterior trunk (Figure 1).

After the end of the acquisition, a hand-held gamma probe is used to precisely locate the skin projection of the SLN, and a skin mark is drawn. During the surgery, a skin incision is made directly over the skin mark and the SLN is explored using the gamma probe (Chakera $\mathrm{AH})$. In some patients, two or more SLNs are identified in different basins; all of them have to be excised and sent for biopsy (Figure 1).

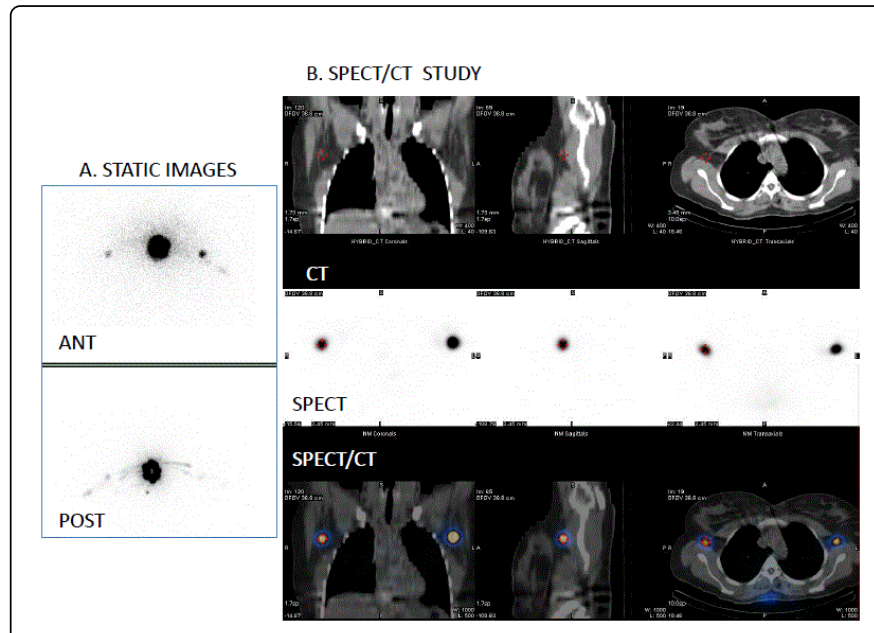

Figure 1: Sentinel lymph node mapping of a patient with malignant melanoma of the posterior upper trunk (right back). Static images (A., anterior and posterior) and B. coronal CT, SPECT and fused SPECT/CT images obtained after intradermal injection of $99 \mathrm{mTc}$ radiocolloid around the melanoma lesion. Static images reveal two drainage pathways of the melanoma lesion and two positive nodes. On SPECT/CT images it is clear that there are two sentinel lymph nodes, one in the right axilla and the other in the left axilla (Nuclear Medicine Department of Patras University Hospital).

\section{Malignant Melanoma Staging}

As in all cancers, accurate initial staging is essential for developing the appropriate treatment strategy. According to the American Joint Committee on Cancer (AJCC), staging of melanoma is based on the TNM (Tumor, Node, Metastasis) system [4]. The mainstay of melanoma staging is wide surgical excision of the primary lesion for pathologic analysis and sentinel lymph node biopsy (SLNB). In melanoma, imaging is focused on detecting occult sites of regional or distant disease.

\section{Indications for SLNB in Malignant Melanoma}

Sentinel lymph node biopsy (SLNB) is a minimally invasive procedure used to accurately stage nodal basins at risk for harboring occult metastases in patients without clinical spread of the disease that has high sensitivity and specificity.

Experts generally agree that patients diagnosed with melanoma in clinical stage T1b-T4b, N0 and M0 should be given an option of SNB [5]. More specifically SLNB is not recommended for primary 
melanomas $<0.75 \mathrm{~mm}$ thick, unless there is significant uncertainty about the adequacy of microstaging (such as ulceration, high mitotic rate and lymphovascular invasion) [3-6]. For melanomas $0.76-1 \mathrm{~mm}$ thick, SLNB should be considered in the appropriate clinical context to determine the presence or absence of regional lymph node metastases, especially when high risk features exist (ulceration, high mitotic rate) [3-6].There is a consensus for SLNB in patients with intermediatethickness melanomas $(1-4 \mathrm{~mm})$ of any anatomic site as it provides accurate staging [3-6]. SLNB may also be considered for patients with resectable solitary in-transit stage III disease (category 2B recommendation) [5]. Although there are few studies focusing on patients with thick melanomas $(>4 \mathrm{~mm})$, that by definition have a high risk of distant metastases, as well as of lymph node metastases (although these are usually not clinically palpable), SLNB still offers very good prognostic information and may be recommended for staging purposes and to facilitate regional disease control [3-6]. However, while SLNB is a useful staging tool, its impact on the overall survival of these patients remains unclear.

\section{Role of PET-CT in the Primary Staging of Malignant Melanoma}

MM is among the most FDG tracer avid cancers known [7]. $\mathrm{PET} / \mathrm{CT}$ has no role in $\mathrm{T}$ staging as it is achieved by histopathologic examination of a full-thickness biopsy of a suspicious lesion. Regarding lymph node status ( $\mathrm{N}$ staging), FDG PET cannot detect micrometastasis, so it cannot replace SLNB in the staging of regional lymph nodes. The capacity of FDG PET to detect lymph nodes metastases is volume dependant in normal sized lymph nodes; metastatic volume equal to or greater than $78 \mathrm{~mm} 3$, while the sensitivity falls rapidly below this volume. Also this amount of tumor is most likely to occur in patients with AJCC stage III or IV disease [8]. A metaanalysis on the diagnostic performance of PET/CT for $\mathrm{N}$ staging in melanoma patients reported a very low sensitivity and high specificity ( $11 \%$ and $97 \%$, respectively) [9].

According to the revised U.K. guidelines (2010) and National Comprehensive Cancer Network (NCCN) guidelines (version 2.2014) for the management of cutaneous melanoma, routine imaging (CT, MRI or PET/CT) is not recommended in asymptomatic patients with stage I and II melanoma (any T,N0,M0) [10]. NCCN guidelines (version 2.2014) add that in patients with stage I or II, imaging (CT, MRI or PET/CT) should be considered, only to evaluate specific signs and symptoms [5].

If SLNB turns to be positive (stage IIIA), these patients will return to the operating room for a completion lymph node dissection. Therefore a question arises whether obtaining imaging at this juncture might be worthwhile. Studies in asymptomatic patients of stage IIIA, indicate a limited role of imaging (CT, MRI, PET or PET/CT) in this group, as it does not reveal occult metastasis and does not alter subsequent melanoma management [11-13] (Figure 2). Constantinidou et al. performed either PET scan or PET/CT in 30 patients with a positive SLNB and a melanoma of Breslow depth greater than $1 \mathrm{~mm}$ and found that only 2 patients had positive findings and neither was melanoma related. NCCN guidelines (version 2.2014) proposes that baseline imaging with CT, PET/CT or MRI should be considered for staging (category 2B) in stage IIIA patients, especially in those with ulcerated thick primary tumors and with large tumor burden in their sentinel nodes. Also baseline imaging with CT, $\mathrm{PET} / \mathrm{CT}$ or MRI should be considered in stage IIIA symptomatic patients to evaluate specific signs and symptoms, before planning regional lymph node dissection and regional chemotherapy [5] (Figure 3).
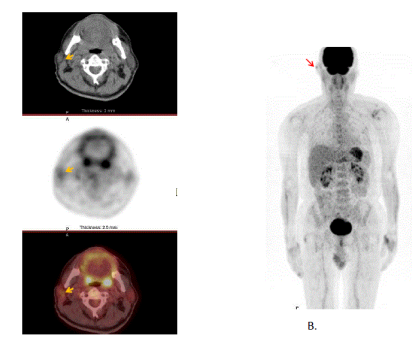

Figure 2: Malignant Melanoma of the right external ear (surgically excised 2 months prior to PET/CT scan) with positive SLNB (stage IIIA). Initial staging. FDG PET/CT study: axial sections of CT, PET and PET/CT (A), as well as Maximum Intensity Projection (MIP) (B) of the attenuation corrected PET study are shown. A focus of FDG tracer uptake is present in the right ear (red arrow) (SUVmax $=2.1$ ) due to postoperative inflammatory changes at the site of resection. There is a very small focus of tracer uptake corresponding to a right intra-parotid lymph node (yellow arrow) $($ SUVmax $=2.0)$. The lymph node was negative on needle biopsy, consistent with a reactive inflammatory lymph node (PET/CT department of the Biomedical Research Foundation of the Academy of Athens).
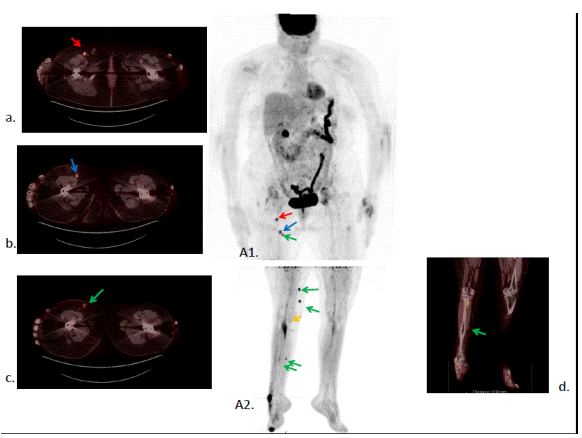

Figure 3: Patient with malignant melanoma in the right thigh (surgically excised 1 month prior to PET/CT scan) and positive right inguinal SLNB (stage IIIA). Initial staging. FDG PET/CT study: axial and coronal sections of PET/CT, as well as Maximum Intensity Projection (MIP) of whole body (A1) and feet (A2) are shown. There is increased FDG uptake in right inguinal (a., A1., red arrow) and right thigh lymph nodes (b. A1., blue arrow) $(\mathrm{SUVmax}=5,9)$ consistent with lymph node metastases. Increased FDG uptake (SUVmax $=4.6$ ) is also noted in multiple subcutaneous nodules in the right thigh and right lower leg (c., d., A2., green arrows), consistent with metastatic subcutaneous deposits. Total hip arthroplasty (THA) bilaterally with subtle diffuse uptake of FDG. The yellow arrow on the MIP image of the feet points the blood pool activity within the popliteal vessels. Increased FDG uptake in the gastrointestinal track due to metformin medication is also noted (PET/CT department of the Biomedical Research Foundation of the Academy of Athens). 
In patients with clinically positive lymph nodes (stage IIIB, IIIC), NCCN guidelines (version 2.2014) proposes that baseline imaging (CT, PET/CT or MRI) is recommended to complete initial staging and to evaluate specific signs and symptoms [5]. In a study by Tyler et al. [14] among 95 patients with clinically evident lymph nodes and/or intransit melanoma, PET revealed 36 of the total 183 abnormal areas (19.7\%) that proved to be unsuspected metastatic sites and led to a change in the planned clinical management in $15 \%$ of cases.

When stage IV disease is suspected clinically or is established, $\mathrm{PET} / \mathrm{CT}$ may have a role when metastasectomy is planned, in excluding disease that might make surgery inappropriate $[5,10]$.

These patients may benefit from the identification of unsuspected or additional distant metastases with subsequent change in management by means of additional surgical resection or avoidance of unnecessary procedures. PET/CT should complement conventional CT and MRI imaging in the staging work-up of patients who have solitary or oligometastatic disease where surgical resection is most relevant [15].

Due to the very high avidity of MM for FDG, PET is a sensitive imaging technique in the detection of metastatic melanoma (Mstaging) and offers several potential advances over CT, including broader field of imaging and higher sensitivity for small lesions in locations other than the brain and the lungs $[16,17]$ particularly in lymph nodes, subcutaneous and cutaneous deposits, soft tissue, visceral organs, small bowel, mesentery and bone metastases $[18,19]$.

According to a recent meta-analysis, based on 10,528 patients between 1990 and 2009, PET/CT was superior to CT, ultrasound and MRI for the detection of distant metastases, whereas ultrasonography was superior for detecting lymph node metastases in the staging of melanoma patients [9].

A number of studies have demonstrated that PET/CT findings affect surgical decision making (from 12 to $49 \%$ ) in patients with melanoma being considered for operative intervention [14,20-26]. In a recent prospective study by Bronstein et al. [26], PET/CT revealed new findings and changed surgical management in 4 out of 33 (12\%) high risk (stage III, IV) but surgically treatable oligometastatic melanoma patients according to conventional imaging (the surgery was canceled for two patients, and the planned approach was altered for another two patients to address the unexpected sites).

In an older prospective study by Gulec et al. [20] forty-nine patients with known or suspected metastatic melanoma, underwent extend of disease evaluation using conventional imaging methods (CT of the chest, abdomen, and pelvis, and MRI of the brain) and whole body PET/CT, after formulation of an initial treatment plan. The results of PET led to surgical and medical treatment alterations in 24 patients (49\%) [20]. All of these studies, however, found that false positive findings were still significant.

Therefore histologic confirmation of suspected metastases must be performed before the treatment plan is altered. The recently introduced combined PET/MR systems will take advantage from superior soft tissue contrast and is expected to help in the more accurate characterization of brain, liver and bone lesions [26].

Overall, PET/CT is more accurate than CT or MRI alone in the diagnosis of metastasis, but there is no convincing evidence that the improved accuracy of PET/CT over CT scanning alone improves patient outcome [27].
In a recent study that systematically reviewed the potential patient relevant benefit of PET and PET/CT in primary staging of malignant melanoma, no randomized control trials were found, and there was no evidence of patient relevant benefit of PET/CT in the primary staging of malignant melanoma.

Diagnostic and prognostic accuracy of PET/CT appears to increase with higher stage. In particular, for patients with stages I and II, sensitivity mostly ranged from 0 to $67 \%$ and specificity ranged from 77 to $100 \%$. For AJCC stages III and IV, sensitivity ranged from 68 to $87 \%$ and specificity from 92 to $98 \%$. However, higher sensitivity and specificity of PET/CT in stages III and IV do not necessarily imply that there is a patient-relevant benefit of PET/CT in this subgroup. Randomized control trials investigating patient relevant outcomes are therefore required [28].

In concluding the role of PET/CT in initial staging of cutaneous melanoma, the most common indications are for patients with locoregional disease at high risk for distant metastases including patients with in-transit and regional lymph node metastases and suspicious clinical and laboratory findings, where identification of additional regional disease would alter planned surgical strategy. Also $\mathrm{PET} / \mathrm{CT}$ should complement conventional CT/MRI imaging in the staging work-up of patients who have solitary or oligometastatic disease where surgical resection is most relevant.

The role of PET/CT in clinically suspicious stage II patients $(>\mathrm{T} 3 \mathrm{~b})$ is evolving rapidly. In addition, PET/CT may be used for evaluation of suspicious or equivocal findings identified by conventional imaging.

\section{Role of PET-CT in Surveillance and Restaging of Melanoma}

The risk of tumor recurrence in malignant melanoma depends on the primary stage and is greatest in the initial years after diagnosis [29]. It has been estimated that $20 \%$ of all first recurrences occur locally around the melanoma scar, 50\% occur in the regional lymph nodes, and $30 \%$ arise at distant sites most commonly the lungs, liver, brain, bone, and gastrointestinal tract [30,31].

The major goal in the follow up of these patients is the early detection of locoregional relapses and limited distant metastatic recurrences that may be amendable to complete resection by salvage surgical therapy. However, an impact of radiological exams upon survival has not been demonstrated so far [32].

According to the Comprehensive Cancer Network guidelines (version 2.2014) for follow-up, in stages IA-IIA no routine radiological imaging to screen for asymptomatic recurrent/metastatic disease is recommended $[5,10,33]$.

For stages IIB-IV, e.g. those with thick primary tumors or following treatment of metastases, or to investigate specific signs and symptoms, apart from common follow-up recommendations (clinical and self examination), ultrasound of lymph nodes, computed tomography (CT) or whole body PET-CT scans (performed periodically for the first 5 years), may lead to an earlier diagnosis of regional or systemic relapses [33] and should be considered to screen for recurrent/ metastatic disease (category 2B) [5] (Figures 4 and 5). 


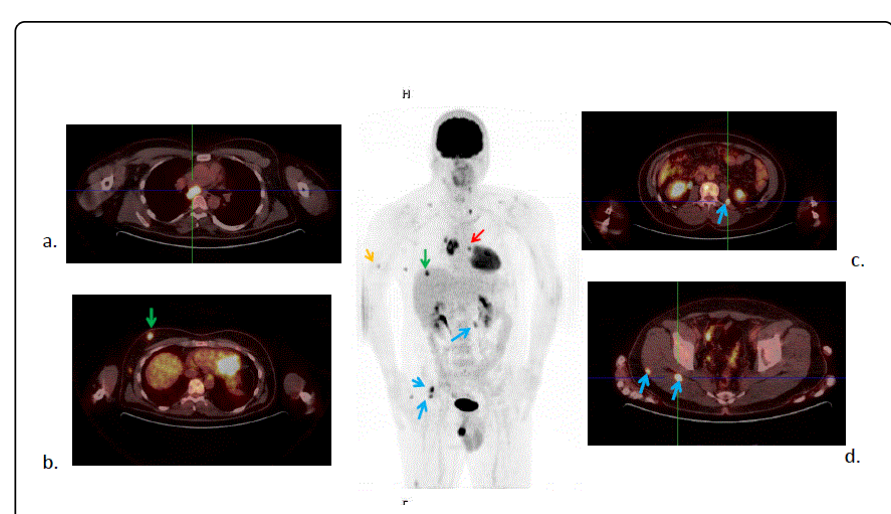

Figure 4: Patient with malignant melanoma of the left posterior auricular region (stage IIIB), surgically treated 5 months ago. Restaging. FDG PET/CT study: transverse sections of PET/CT, as well as Maximum Intensity Projection (MIP) of the attenuation corrected PET study are shown. Multiple sites of metastasis are noted in cervical lymph nodes, in mediastinal lymph nodes (subcarinal and right hilum) (a.), in a left lung nodule (red arrow), in soft tissues (c, d, blue arrows) and in multiple cutaneous and subcutaneous nodules of the thoracic wall (b) (one of these nodules, is projecting over the liver on the MIP image, green arrow) and right upper arm (yellow arrow) (PET/CT department of the Biomedical Research Foundation of the Academy of Athens).

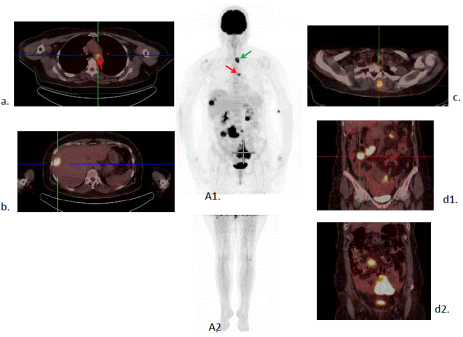

Figure 5: Patient with malignant melanoma (stage IIIB) of the left upper arm, surgically treated 5 years ago. Metastasis in small bowel, surgically treated 3 years ago. Restaging. FDG PET/CT study: Axial and coronal sections of PET/CT, as well as Maximum Intensity Projection (MIP) of whole body (A1) and feet (A2) are shown. Multiple sites of metastases are noted in a mediastinal lymph node (a., A/P window, red arrow), in the liver (b), in the abdomen (peritoneum and small bowel involvement) and in rhomboideus major muscle (c., green arrow) (PET/CT department of the Biomedical Research Foundation of the Academy of Athens).

In a recent study by Abbott et al. [3], thirty-four patients with stage III melanoma were followed at least annually with PET/CT. In twenty patients with stage IIIA disease at diagnosis, annual surveillance $\mathrm{PET} / \mathrm{CT}$ detected two out of three recurrences while in fourteen patients with stage IIIB and IIIC disease at diagnosis, annual PET/CT detected four out of four recurrences, and distant metastases in one asymptomatic patient. Although the numbers in this study are small, the role of PET/CT in patients initially presenting with stage III disease warrants further confirmation [34]. In another study by Beasley et al. [35] FDG-PET/CT performed every 3 months after treatment, appears to be an excellent method for surveillance in stage IIIB/IIIC patients with extremity melanoma, with ability to identify surgically resectable recurrent disease in these high-risk patients.

According to the results of a recent metaanalysis, the positive predictive value (PPV) of $\mathrm{PET} / \mathrm{CT}$ for the detection of recurrent metastases is stage-dependent, yielding a higher PPV in high-risk patients $(80 \%)$ than in intermediate-risk patients $(63 \%)$ and low-risk patients (33\%) [9]. Overall PET-CT was superior to CT for the detection of distant metastases in surveillance of melanoma patients [9].

A retrospective study in high-risk melanoma patients found that FDG PET/CT was more accurate for the detection of tumor recurrence than the tumor marker $\mathrm{S} 100$, that is considered the most accurate blood test in the follow-up of melanoma patients [36,37]. The same study by Essler et al. revealed that PET/CT, compared with S100, had a significantly higher prognostic value for cancer-related mortality.

When a recurrence is observed or confirmed by biopsy (local recurrence, satellite, in-transit, nodal or distant metastasis), baseline imaging (CT, PET/CT, MRI) is recommended for restaging and to evaluate specific signs and symptoms (category 2B) (NCCN). For example, in patients with locoregional recurrent disease (lesions at the primary melanoma site, satellite lesions less than $2 \mathrm{~cm}$ from the primary melanoma site, in-transit metastases, and involved regional lymph nodes) PET-CT is helpful to determine the presence or absence of distant metastatic disease and fully characterize the locoregional recurrence.

In patients with suspected recurrent melanoma, PET/CT has been shown to influence treatment planning from $27 \%$ [24,38] to $40 \%$ [39]. Etchebehere et al. [38] reviewed seventy-eight patients with suspected melanoma recurrence and found that in $27 \%$ of the patients the management was changed after the FDG PET/CT studies regarding planned surgical, radiotherapy, or chemotherapy. Upstaging from locoregional recurrence to distant recurrence occurred in 5 out of 23 patients (22\%) and PET showed 95\% sensitivity for detecting metastatic lesions [38]. False-positive findings remains a concern for this application and histologic confirmation or further imaging investigation of suspected metastases must be performed before the treatment plan is altered.

For patients with known extensive distant metastatic disease, $\mathrm{PET} / \mathrm{CT}$ would add little to patient management, except perhaps as baseline inventory for the metastatic burden prior to systemic therapy. In patients with known distant metastatic disease that appears limited, and resection of the metastasis is considered, PET-CT is useful to exclude additional unsuspected metastases.

In concluding the role of $\mathrm{PET} / \mathrm{CT}$ in restaging of cutaneous melanoma the most common indications are for patients with locoregional relapse and/or isolated distant metastatic recurrence under consideration for surgical resection. The use of PET/CT in patients with high risk locoregional disease undergoing surveillance after initial treatment has shown great promise.

\section{Treatment Response Evaluation in Malignant Melanoma}

Although stage IV melanoma is an incurable disease with significant chemoresistance, new immunotherapeutic agents have been tested such as interferon (INF), interleukin-2 (IL-2), denileukin/ diftitox (DAB/IL2; that is a recombinant fusion protein product of 
diphtheria toxin and IL-2), ipilimumab (an anti-cytotoxic T lymphocyte antigen-4 [CTLA4] antibody), selective BRAF inhibitors like vemurafenib and dabrafenib, c-Kit inhibitors, MAPK/ERK kinase (MEK) inhibitors, and dendritic cell vaccination [40-42]. Assessing response of melanoma to immunotherapeutic agents is becoming increasingly important and FDG PET/CT can aid in evaluating clinical efficacy of these new agents. In a recent single-center, phase II trial, by Telang et al. the clinical efficacy of DAB/IL2 in an expanded cohort (60 patients) of stage IV melanoma patients was assessed using a combination of FDG PET/CT imaging. Baseline FDG PET/CT imaging was compared to follow-up scans using qualitative radiology assessments which approximate Response Evaluation Criteria in Solid Tumors (RECIST) criteria. The results showed that DAB/IL2 has significant clinical activity against stage IV melanoma and patients who respond live significantly longer than patients who experience progressive disease. Based on the results of this study, the development of multi-center, randomized trials of DAB/IL2 as a monotherapy and in combination with other immunotherapeutic agents for the treatment of stage IV melanoma is supported [43].

In another phase I study by McArthur et al. [44] baseline and day 15 FDG-PET was evaluated in 31 patients with BRAF-Mutant advanced MM treated with dose escalation of vemurafenib (PLX06-02). The results showed that all patients treated at potentially therapeutic levels had at least a partial metabolic response (PMR), and three patients achieved a complete metabolic response (CMR). There was a positive correlation between the percentage of injected dose and reduction in the maximum standardized uptake value (SUVmax) of target lesions that indicated a significant homogeneity of the response between lesions in individual patients. Although no relationship was found between the reduction in target lesion SUVmax and best response according to RECIST, there was a trend for patients with greater reductions in uptake of FDG to have longer progression-free survival.

In a recent retrospective study from Engell-Noerregaard et al. [45], thirteen patients with advanced progressive MM were treated with dendrtitic cell vaccinations and evaluated by PET/CT scans at baseline as well as after 6 vaccinations. According to RECIST, three patients achieved stable disease (SD) and ten patients progressed. Interestingly, one out of three patients with SD (according to CT scan) was found to have progressive metabolic disease (PMD) according to the PET scan due to a new lesion appearing after 6 vaccinations, while two other SD patients achieved PMR.

From the aforementioned studies, it is clear that further investigation into the contribution of PET scans to the evaluation of MM immunotherapy is needed.

\section{Selected Issues in Performing and Reporting PET-CT of Melanoma}

Due to the propensity of malignant melanoma to metastasize in widespread and unpredictable patterns, the PET-CT imaging protocols for staging and restaging melanoma require extensive axial coverage, usually the whole body, from the skull vertex to the bottom of the feet, rather than the more typical "eyes to thighs" scan done for other oncologic purposes. In a recent retrospective study by Lazaga et al. [46], of 200 patients who underwent ${ }^{18}$ F-FDG PET/CT for staging melanoma, whole-body PET/CT scans including the lower extremities were reviewed and the results showed that in patients with malignant cutaneous melanoma that did not arise in the lower extremities, additional lower-extremity scan could be discontinued with a high degree of confidence. PET/CT acquisition of the lower extremities in these patients, adds no useful information whereas it results in increased scan time and unnecessary extra radiation dose from CT.

The use of intravenous and oral contrast agents in routine PET/CT protocol for melanoma have not been demonstrated to add significant effect [47], whereas they directly affect SUVs and the safety profile of the study (for the iv contrast agents).

In the interpretation of the PET/CT scan it is important to know the extent of excision and postoperative inflammatory changes at the primary site and the interval between biopsy and the PET study, as false-positive lymph node findings will be increased in the setting of inflammatory changes at the site of surgical resection.

\section{Conclusion}

The role of nuclear medicine in the evaluation and management of patients with skin cancer is centered mainly in malignant melanoma because of its aggressive features and its FDG avidity. SLNB is the mainstay in melanoma staging while the role of PET/CT even in earlier stages is evolving rapidly.

PET/CT combines the advantages of the metabolic imaging and anatomic localization in one session. PET/CT with its inherent whole body capability is arguably the best imaging option to search for melanoma metastases with the potential to change treatment plan in as high as $49 \%$. Currently PET/CT is most useful for identifying all metastatic sites of disease before embarking on a metastasectomy of an apparently isolated lesion (stage IV patients) or for patients at high risk for distant metastases based on the extent of locoregional disease at diagnosis (stage III patients). Also clinically suspicious stage II patients (>T3b) are felt to be suitable for staging with PET/CT. $\mathrm{PET} / \mathrm{CT}$ is also employed in restaging patients before the removal of presumed solitary metastases or in the confirmation of recurrent disease and in clarification of the nature of a suspicious lesion identified by the CT scan.The use of PET/CT in surveillance for relapse has shown promise, but it is not yet widely practiced. Hopefully with future advances intreatment options, the role of $\mathrm{PET} / \mathrm{CT}$ would become more defined in the assessment of response to melanoma therapy.

\section{References}

1. Phelps ME, Hoffman EJ, Huang SC, Ter-Pogossian MM (1975) Effect of positron range on spatial resolution. J Nucl Med 16: 649-652.

2. Buchbender C, Heusner TA, Lauenstein TC, Bockisch A, Antoch G (2012) Oncologic PET/MRI, part 2: bone tumors, soft-tissue tumors, melanoma, and lymphoma. J Nucl Med 53: 1244-1252.

3. Chakera AH, Hesse B, Burak Z, Ballinger JR, Britten A, et al. (2009) EANM-EORTC general recommendations for sentinel node diagnostics in melanoma. Eur J Nucl Med Mol Imaging 36: 1713-1742.

4. Balch CM, Gershenwald JE, Soong SJ, Thompson JF, Atkins MB, et al. (2009) Final version of 2009 AJCC melanoma staging and classification. J Clin Oncol 27: 6199-6206.

5. http://www.nccn.org/professionals/physician_gls/def/ault.asp.

6. Wong SL, Balch CM, Hurley P, Agarwala SS, Akhurst TJ, et al. (2012) Sentinel lymph node biopsy for melanoma: American Society of Clinical Oncology and Society of Surgical Oncology joint clinical practice guideline. J ClinOncol 30: 2912-2918.

7. Wahl RL, Hutchins GD, Buchsbaum DJ, Liebert M, Grossman HB, et al. (1991) 18F-2-deoxy-2-fluoro-D-glucose uptake into human tumor xenografts. Feasibility studies for cancer imaging with positron-emission tomography. Cancer 67: 1544-1550. 
8. Wagner JD, Schauwecker DS, Davidson D, Wenck S, Jung SH, et al. (2001) FDG-PET sensitivity for melanoma lymph node metastases is dependent on tumor volume. J Surg Oncol 77: 237-242.

9. Xing Y, Bronstein Y, Ross MI, Askew RL, Lee JE, et al. (2011) Contemporary diagnostic imaging modalities for the staging and surveillance of melanoma patients: a meta-analysis. J Natl Cancer Inst 103: 129-142.

10. Marsden JR, Newton-Bishop JA, Burrows L, Cook M, Corrie PG, et al. (2010) Revised U.K. guidelines for the management of cutaneous melanoma 2010. Br J Dermatol 163: 238-256.

11. Clark PB, Soo V, Kraas J, Shen P, Levine EA (2006) Futility of fluorodeoxyglucose F 18 positron emission tomography in initial evaluation of patients with T2 to T4 melanoma. Arch Surg 141: 284-288.

12. Constantinidou A, Hofman M, O'Doherty M, Acland KM, Healy C, et al. (2008) Routine positron emission tomography and positron emission tomography/computed tomography in melanoma staging with positive sentinel node biopsy is of limited benefit. Melanoma Res 18: 56-60.

13. Wagner T, Meyer N, Zerdoud S, Julian A, Chevreau C, et al. (2011) Fluorodeoxyglucose positron emission tomography fails to detect distant metastases at initial staging of melanoma patients with metastatic involvement of sentinel lymph node. Br J Dermatol 164: 1235-1240.

14. Tyler DS, Onaitis M, Kherani A, Hata A, Nicholson E, et al. (2000) Positron emission tomography scanning in malignant melanoma. Cancer 89: 1019-1025.

15. Mohr P, Eggermont AM, Hauschild A, Buzaid A (2009) Staging of cutaneous melanoma. Ann Oncol 20 Suppl 6: vil4-21.

16. Friedman KP, Wahl RL (2004) Clinical use of positron emission tomography in the management of cutaneous melanoma. Semin Nucl Med 34: 242-253.

17. Segall GM, Swetter SM, Townsend DW, Maisey MN (2003) Positron Emission Tomography. Basic Science and Clinical Practice. London: Springer-Verlag 2004: 884.

18. Swetter SM, Carroll LA, Johnson DL, Segall GM (2002) Positron emission tomography is superior to computed tomography for metastatic detection in melanoma patients. Ann Surg Oncol 9: 646-653.

19. Aydin A, Yu JQ, Zhuang H, Alavi A (2005) Detection of bone marrow metastases by FDG-PET and missed by bone scintigraphy in widespread melanoma. Clin Nucl Med 30: 606-607.

20. Gulec SA, Faries MB, Lee CC, Kirgan D, Glass C, et al. (2003) The role of fluorine-18 deoxyglucose positron emission tomography in the management of patients with metastatic melanoma: impact on surgical decision making. Clin Nucl Med 28: 961-965.

21. Brady MS, Akhurst T, Spanknebel K, Hilton S, Gonen M, et al. (2006) Utility of preoperative [(18)]f fluorodeoxyglucose-positron emission tomography scanning in high-risk melanoma patients. Ann Surg Oncol 13: 525-532.

22. Harris MT, Berlangieri SU, Cebon JS, Davis ID, Scott AM (2005) Impact of 2-deoxy-2[F-18]fluoro-D-glucose Positron Emission Tomography on the management of patients with advanced melanoma. Mol Imaging Biol 7: 304-308.

23. Reinhardt MJ, Joe AY, Jaeger U, Huber A, Matthies A, et al. (2006) Diagnostic performance of whole body dual modality 18F-FDG PET/CT imaging for $\mathrm{N}$ - and $\mathrm{M}$-staging of malignant melanoma: experience with 250 consecutive patients. J Clin Oncol 24: 1178-1187.

24. Falk MS, Truitt AK, Coakley FV, Kashani-Sabet M, Hawkins RA, et al. (2007) Interpretation, accuracy and management implications of FDG PET/CT in cutaneous malignant melanoma. Nucl Med Commun 28: 273-280.

25. Pfannenberg C, Aschoff P, Schanz S, Eschmann SM, Plathow C, et al. (2007) Prospective comparison of 18F-fluorodeoxyglucose positron emission tomography/computed tomography and whole-body magnetic resonance imaging in staging of advanced malignant melanoma. Eur J Cancer 43: 557-564.

26. Bronstein Y, Ng CS, Rohren E, Ross MI, Lee JE, et al. (2012) PET/CT in the management of patients with stage IIIC and IV metastatic melanoma considered candidates for surgery: evaluation of the additive value after conventional imaging. AJR Am J Roentgenol 198: 902-908.

27. Iagaru A, Quon A, Johnson D, Gambhir SS, McDougall IR (2007) 2Deoxy-2-[F-18]fluoro-D-glucose positron emission tomography/ computed tomography in the management of melanoma. Mol Imaging Biol 9: 50-57.

28. Schröer-Günther MA, Wolff RF, Westwood ME, Scheibler FJ, Schürmann C, et al. (2012) F-18-fluoro-2-deoxyglucose positron emission tomography (PET) and PET/computed tomography imaging in primary staging of patients with malignant melanoma: a systematic review. Syst Rev 1: 62 .

29. Slingluff CL Jr, Dodge RK, Stanley WE, Seigler HF (1992) The annual risk of melanoma progression. Implications for the concept of cure. Cancer 70: 1917-1927.

30. Benvenuto-Andrade C, Oseitutu A, Agero AL, Marghoob AA (2005) Cutaneous melanoma: surveillance of patients for recurrence and new primary melanomas. Dermatol Ther 18: 423-435.

31. Meier F, Will S, Ellwanger U, Schlagenhauff B, Schittek B, et al. (2002) Metastatic pathways and time courses in the orderly progression of cutaneous melanoma. Br J Dermatol 147: 62-70.

32. Nieweg OE, Kroon BB (2006) The conundrum of follow-up: should it be abandoned? Surg Oncol Clin N Am 15: 319-330.

33. Dummer R, Hauschild A, Guggenheim M, Keilholz U, Pentheroudakis G; ESMO Guidelines Working Group (2012) Cutaneous melanoma: ESMO Clinical Practice Guidelines for diagnosis, treatment and follow-up. Ann Oncol 23 Suppl 7: vii86-91.

34. Abbott RA, Acland KM, Harries M, O'Doherty M (2011) The role of positron emission tomography with computed tomography in the followup of asymptomatic cutaneous malignant melanoma patients with a high risk of disease recurrence. Melanoma Res 21: 446-449.

35. Beasley GM, Parsons C, Broadwater G, Selim MA, Marzban S, et al. (2012) A multicenter prospective evaluation of the clinical utility of F-18 FDG-PET/CT in patients with AJCC stage IIIB or IIIC extremity melanoma. Ann Surg 256: 350-356.

36. Beyeler M, Waldispuhl S, Strobel K, Joller-Jemelka HI, Burg G, et al. (2006) Detection of melanoma relapse: first comparative analysis on imaging techniques versus S100 protein. Dermatology 213: 187-191.

37. Essler M, Link A, Belloni B, Mirceva V, Souvatzoglou M, et al. (2011) Prognostic value of [18F]-fluoro-deoxy-glucose PET/CT, S100 or MIA for assessment of cancer-associated mortality in patients with high risk melanoma. PLoS One 6: e24632.

38. Etchebehere EC, Romanato JS, Santos AO, Buzaid AC, Camargo EE (2010) Impact of [F-18] FDG-PET/CT in the restaging and management of patients with malignant melanoma. Nucl Med Commun 31: 925-930.

39. Mijnhout GS, Comans EF, Raijmakers P, Hoekstra OS, Teule GJ, et al. (2002) Reproducibility and clinical value of 18F-fluorodeoxyglucose positron emission tomography in recurrent melanoma. Nucl Med Commun 23: 475-481.

40. Wolchok JD, Neyns B, Linette G, Negrier S, Lutzky J, et al. (2010) Ipilimumab monotherapy in patients with pretreated advanced melanoma: a randomised, doubleblind, multicentre, phase 2, doseranging study. Lancet Oncol 11: 155-164.

41. Foss FM1 (2000) DAB(389)IL-2 (denileukin diftitox, ONTAK): a new fusion protein technology. Clin Lymphoma 1 Suppl 1: S27-31.

42. Flaherty KT, Robert C, Hersey P, Nathan P, Garbe C, et al. (2012) Improved survival with MEK inhibition in BRAF-mutated melanoma. N Engl J Med 367: 107-114.

43. Telang S, Rasku MA, Clem AL, Carter K, Klarer AC, et al. (2011) Phase II trial of the regulatory $\mathrm{T}$ cell-depleting agent, denileukin diftitox, in patients with unresectable stage IV melanoma. BMC Cancer 11: 515.

44. McArthur GA, Puzanov I, Amaravadi R, Ribas A, Chapman P, et al. (2012) Marked, Homogeneous, and Early [18F]Fluorodeoxyglucoseâ€ "Positron Emission Tomography Responses to Vemurafenib in BRAFMutant Advanced Melanoma. J ClinOncol 30: 1628-1634. 
Citation: Paschali AN (2015) The Role of Nuclear Medicine in Malignant Melanoma. J Nucl Med Radiat Ther 6: 237. doi: $10.4172 / 2155-9619.1000237$

Page 8 of 8

45. Engell-Noerregaard L, Hendel HW, Johannesen HH, Alslev L, Svane IM (2013) FDG PET scans as evaluation of clinical response to dendritic cell vaccination in patients with malignant melanoma. Cancer Immunol Immunother 62: 17-25.

46. Lazaga FJ, Oz OK, Adams-Huet B, Anderson J, Mathews D (2013) Comparison of whole-body versus limited whole-body 18F-FDG
PET/CT scan in malignant cutaneous melanoma. Clin Nucl Med 38: 882-884.

47. Pfluger T, Melzer HI, Schneider V, La Fougere C, Coppenrath E, et al. (2011) PET/CT in malignant melanoma: contrast-enhanced CT versus plain low-dose CT. Eur J Nucl Med Mol Imaging 38: 822-831. 